Revista de la Escuela de Ciencias de la Educación, año 13, nRo. 12, vol. 2, julio a diciembre de 2017. Páginas 67-86. ISSN 1851-6297. ISSN 2362-3349 (EN LINEA). EL SISTEMA DE FORMACIÓN DOCENTE Y LA PRODUCCIÓN, RELEVANCIA, VALIDACIÓN y Legitimación del Conocimiento. Algunas dimensiones para abordar una agenda aún pendiente. Cristina Rafaela Ricci

\title{
EL SISTEMA DE FORMACIÓN DOCENTE Y LA PRODUCCIÓN, RELEVANCIA, VALIDACIÓN Y LEGITIMACIÓN DEL CONOCIMIENTO: ALGUNAS DIMENSIONES PARA ABORDAR UNA AGENDA AÚN PENDIENTE
}

\author{
Por Cristina Rafaela Ricci* \\ ISFD N 41 y UNLP Buenos Aires, Argentina. \\ crcristinaricci@yahoo.com.ar
}

Recibido: 30/09/2016 Aceptado: 01/11/2016

\section{Resumen}

Analizar cómo, dónde, quiénes y a través de qué procedimientos se valida, legitima y difunde el conocimiento producido por los Institutos de Educación Superior (IES), requiere pensar más allá de los contextos clásicos de descubrimiento y justificación e, incluso, ir más allá de la concepción moderna de ciencia. En este sentido, sostengo que los planteos epistemológicos y metodológicos de la concepción heredada de la modernidad, no son los más aptos para otorgar estatus científico a las producciones de los IES.

Surgen así, por lo menos dos dimensiones a tener en cuenta al momento de abordar algunas preguntas centrales que, el Sistema de Formación Docente debe asumir como parte de su agenda: una dimensión político-educativa y otra espistemológico-metodológica.

Magister y Especialista en Metodología de la Investigación Científica (UNLa). Especialista en Nuevas Infancias y Juventudes (UNQ), Especialista en Investigación Educativa (UTN), Especialista en Educación Superior y TIC (INFD), Profesora y Licenciada en Ciencias de la Educación (UNLZ-UBA), Licenciada y Psicopedagoga (UNLZ), Profesora Especializada en Educación Especial (ISFD N 18), Asistente Educacional (ISFD $N^{\circ}$ 18), Maestra Normal Superior (ISFD N¹1) y Doctoranda en Ciencias de la Educación (UNLP). Se desempeña como Profesora e Investigadora en los ISFD N ${ }^{\circ} 18, N^{\circ} 35 ; N^{\circ} 41$ y N 102 (Buenos Aires). 
Entonces, ¿Desde qué perspectivas epistemológicas y metodológicas hay que analizar las investigaciones que se desarrollan en los IES? ¿Por qué hasta el presente los conocimientos y saberes producidos en los Institutos Superiores de Formación Docente (ISFD) no son reconocidos como tales? La validación, legitimación y relevancia del conocimiento producido por los IES/ISFD, ¿Cómo, quiénes y para qué se establecen? Reflexionar sobre esto puede contribuir a que la producción de saberes y conocimiento se constituya en un rasgo identitario del Sistema de Formación Docente.

Palabras clave: Producción/relevancia - Validación - Legitimación - Conocimiento - Sistema de Formación Docente.

\section{Abstract}

To analyze how, where, who and through which procedures is knowledge produced by the Institutes of Higher Education (IHE) validated, legitimized and communicated, requires thinking beyond the classical contexts of discovery and justification, and even go beyond the modern conception of science. In this sense, I argue that the epistemological and methodological proposals of the inherited conception from modernity are not the most adequate to give scientific status to the productions of the IHE.

Thus, at least two dimensions arise to consider when addressing some key questions that the teacher training system must assume as part of its agenda: a political-educational dimension and another epistemological and methodological.

So from what epistemological and methodological perspectives should the research undertaken in the IES be analyzed? Why is it that to the present time knowledge and what is known produced in the Higher Teacher Training Institutes ( $\mathrm{HTTl})$ are not recognized as such? Validation, legitimization and relevance of the knowledge produced by the IHE / HTTI, how, who and what for are they established? Reflecting on this can contribute to the production of knowledge and what is known constitutes an identifying feature of the System of Teacher Education.

Keywords: Production/relevance - Validation - Legitimization - Knowledge System of Teacher Education.

\section{Artículo}

El presente artículo presenta algunas de las discusiones teóricas y reflexiones que se abren en torno al tema de estudio de la tesis de doctorado en curso: la producción, relevancia del conocimiento producido en Institutos de Educación Superior y, su validación y legitimación por parte de la comunidad académico-científica y por parte de las políticas públicas. La tesis se desarrolla en el marco del Doctorado en Ciencias de la Educación de la Facultad 
Revista de la Escuela de Ciencias de la Educación, año 13, nRo. 12, vol. 2, julio a diciembre de 2017. Páginas 67-86. ISSN 1851-6297. ISSN 2362-3349 (EN LINEA). EL SISTEMA DE FORMACIÓN DOCENTE Y LA PRODUCCIÓN, RELEVANCIA, VALIDACIÓN y Legitimación del Conocimiento. Algunas dimensiones para abordar una agenda aún pendiente. Cristina Rafaela Ricci

de Humanidades y Ciencias de la Educación de la Universidad Nacional de La Plata. La misma tiene como título provisorio Perspectivas epistemológicas y metodológicas emergentes que contribuyan para establecer la validación, legitimación y relevancia de la pertinencia del conocimiento producido en el Sistema de Formación Docente en la República Argentina entre los años 2006-2016.

La perspectiva de la tesis es cualitativa, tratándose de una investigación descriptiva, siendo su propósito, justamente, describir las características del objeto de conocimiento, en este caso los modos de producción, validación y legitimación del conocimiento en los Institutos de Educación Superior (IES), focalizándose en Institutos Superiores de Formación Docente (ISFD). Si bien este tipo de enfoque de investigación no conlleva un alto grado de control de las variables, es decir, no supone la manipulación de variables como se da en los estudios experimentales, sí exige el tratamiento riguroso de los datos obtenidos a partir de un muestreo teórico. El tratamiento de los datos consiste, básicamente, en la selección de casos e informantes, los que se detallan a continuación en relación con las estrategias metodológicas aplicadas, en la medida en que cada uno de los que se adicione arroje nuevos elementos, nueva información. Cuando se llega al punto en que los casos no agregan nueva información, se llega a la saturación teórica (Teoría Fundamentada). Este tipo de muestreo ha sido elegido porque permite realizar un estudio en profundidad (Arroyo, 2011; Pievi, 2009; Soneira, 2009).

La investigación se encuentra en la etapa de sistematización de la información y de construcción de los datos para lo cual se aplica el método comparativo constante (MCC). El MCC permite el análisis comparativo y sistémico de las unidades de análisis, integra los resultados del muestreo teórico y de la saturación teórica para la generación de categorías teorías partiendo, así, la codificación propia del análisis de contenido. Se optó por el MCC porque esta Tesis no busca la prueba de hipótesis, sino que tiene como propósito generar categorías conceptuales y propiedades en relación a su objeto de estudio mediante la comparación constante en la búsqueda de semejanzas y diferencias a través del análisis de los incidentes contenidos en los datos para hallar regularidades (de la Torre, 2008; Bardin, 1996; Vázquez Sixto, 1996).

Para tal fin fueron empleadas las siguientes estrategias metodológicas abarcando el recorte temporal 2006-2016 en la República Argentina: administración de entrevistas y/o cuestionarios auto-administrados a referentes del campo académico universitario, a referentes del campo académico de los ISFD, a referentes del Colectivo Argentino de Educadoras y Educadores que hacen investigación desde la escuela (CA) -colectivo que nuclea distintas redes de docentes y organizaciones sociales-, a referentes del campo de la política educativa encargados de gestionar el área de investigación del Instituto Nacional de Formación Docente (INFD, Ministerio de Educación y Deportes 
de la Nación); encuestas online a nivel nacional a los Directores de Proyectos de investigación de las Convocatorias del INFD entre los años 2007-2014; análisis de fuentes documentales primarias tales como: Informes finales publicados en el Centro de Documentación del INFD (CEDOC) de las Investigaciones de las Convocatorias del INFD 2007-2013; Ponencias presentadas en Encuentros Nacionales del Colectivo Argentino 2006-2016; instructivos del INFD: Bases de las Convocatorias 2007-2015 para la presentación de Proyectos de investigación, Instructivos para la confección de informes finales de las Investigaciones de las Convocatorias del INFD 2007-2015, Instructivos para los evaluadores externos al INFD de Proyectos de Investigación y de Informes finales de las Convocatorias INFD 2007-2015 y, finalmente las Convocatorias para los Encuentros Nacionales del CA 2006-2016, Ponencias presentadas en los distintos ejes en los Encuentros Nacionales del CA 2008-2016 e Instructivos del CA para la realización de Lectura entre pares de ponencias los Encuentros Nacionales del CA 2008-2016.

En este artículo se proponen para el análisis y discusión dos dimensiones que, a mi criterio, deberían ser constitutivas de una agenda, aún pendiente, en la comunidad política y académico-científica: el vínculo entre el Sistema de Formación Docente y la producción, relevancia, validación y legitimación del conocimiento. Estas dimensiones son, la dimensión político-educativa y la dimensión epistemológico-metodológica, que bien podrían articularse sinérgicamente como la dimensión epistemológico-política teniendo en cuenta que el Sistema formador y las instituciones que lo conforman, en tanto parte del Sistema Educativo Argentino, son un subsistema socio-político.

\section{Preliminares}

En cada época histórica se puede reconocer una concepción de saber basada en los criterios que ésta supone de lo que es conocimiento en sentido estricto, es decir, de lo que considera como conocimiento científico. Se trata, tal como lo plantea Rubén Pardo (2000), del saber que cada época considera válido, donde la pregunta ¿qué es ciencia? es una pregunta cuya respuesta varía históricamente, porque la comunidad científica de cada época -de acuerdo con las prácticas sociales y con el modo como esa comunidad comprende la realidad- forja un sentido determinado de ciencia. Ello remite, entonces, a la imposibilidad de sostener la presencia de una estructura fija y única en la construcción de conocimiento.

En este sentido, Edgar Morin (1997) sostiene que el conocimiento científico fue concebido durante mucho tiempo, y aún lo es a menudo, como teniendo por misión disipar la aparente complejidad de los fenómenos a fin de revelar el orden simple al que obedecen. Sin embargo, entiende que lo complejo no puede resumirse en el término complejidad o retrotraerse a una ley de complejidad. La complejidad, efectivamente, 
Revista de la Escuela de Ciencias de la Educación, año 13, nRo. 12, vol. 2, julio a diciembre de 2017. Páginas 67-86. ISSN 1851-6297. ISSN 2362-3349 (EN LINEA). EL SISTEMA DE FORMACIÓN DOCENTE Y LA PRODUCCIÓN, RELEVANCIA, VALIDACIÓN y Legitimación del Conocimiento. Algunas dimensiones para abordar una agenda aún pendiente. Cristina Rafaela Ricci

es el tejido de eventos, acciones, interacciones, retroacciones, determinaciones, azares, que constituyen nuestro mundo fenoménico. Así es que la complejidad se presenta con los rasgos inquietantes de lo enredado, de lo inextricable, del desorden, la ambigüedad, la incertidumbre (...) De allí la necesidad, para el conocimiento, de poner orden en los fenómenos rechazando el desorden, de descartar lo incierto, es decir, de seleccionar los elementos de orden y de certidumbre, de quitar ambigüedad, clarificar, distinguir, jerarquizar (Morin, 1997, p. 32).

Es así que las variantes son múltiples y posibles al momento de conceptualizar al conocimiento al mismo tiempo que permiten desmitificar la validez del pensamiento único y del método universal porque se constata una realidad cada vez más compleja y multidimensional, llena de eventos y acciones, interacciones y retroacciones, que no admite racionalismos reductivos, donde se hace visible la coexistencia de múltiples enfoques, a su vez en tensión, para abordar problemas complejos, tal como lo señala Marcelo Trejo (2010). Del mismo modo Michel Foucault (1981) pone de manifiesto el carácter transitorio e histórico de una teoría científica y plantea la pasividad de un tipo de conocimiento que se ha ido construyendo sobre la irrupción y la discontinuidad y no sobre la acumulación, que asocia el desarrollo de una teoría en función de su evolución precedente desde un origen fundante.

Por lo tanto, cabe preguntarse con Pardo, “¿En qué consiste el saber propio de la ciencia? ¿Qué características ha de tener el conocimiento científico?” (Pardo, 2000, p. 38). La variación epocal de las respuestas a estas preguntas permite, por un lado, analizar el término ciencia en dos sentidos: uno amplio, epocal, diacrónico y otro restringido, actual, sincrónico. Por otro lado, habilitan pensar en otras formas de concebir al conocimiento y las formas de producción, validación, legitimación y relevancia de ese conocimiento producido epocalmente.

En su sentido amplio e histórico puede hablarse, según Pardo, de tres grandes modelos epocales o paradigmas vinculados al modo de comprender al conocimiento científico: un paradigma premoderno que abarca la Antigüedad y la Edad Media (desde el siglo VI antes de Cristo hasta el siglo $\mathrm{XV}$ ); un paradigma moderno, surgido a partir de las revoluciones científicas de los siglos XVI y XVII que se extiende hasta la primera mitad del siglo XX y, un paradigma actual -por algunos llamado posmoderno o tardomoderno por otros- que se constituye fundamentalmente en el $\mathrm{XX}$ y que, si bien no difiere totalmente del de la modernidad, guarda respecto de él muchas e importantes diferencias (Pardo, 2000).

Ahora bien, ¿Cuáles son esas diferencias? ¿Se puede continuar sosteniendo el concepto moderno de ciencia que la entiende como "un tipo de cono- 
cimiento que debe cumplir con ciertos requisitos tales como, capacidad descriptiva, explicativa y predictiva mediante leyes, con carácter crítico, metódico y sistemático; con fundamentación lógica y empírica, comunicable mediante un lenguaje preciso y con pretensión de objetividad?" (Pardo, 2000, p. 42).

Hoy, ¿Por qué surge la necesidad de redefinir al conocimiento científico, sus modos y contextos de producción y validación?

En todo caso, esta pregunta se justifica desde la perspectiva de la Educación Superior si se considera que la Ley de Educación Nacional Argentina (LEN, 2006), establece que la Educación Superior está comprendida a nivel nacional por las Universidades e Institutos Universitarios, estatales o privados, así como por los Institutos de Educación Superior de jurisdicción nacional, provincial o de la Ciudad Autónoma de Buenos Aires, de gestión estatal o privada que tienen, entre otras finalidades la producción de los conocimientos necesarios para la formación integral de las personas, el desarrollo nacional y la construcción de una sociedad más justa. Para tal fin, la LEN crea el Instituto Nacional de Formación Docente (INFD) como organismo responsable, entre otras funciones, de impulsar y desarrollar acciones de investigación en los IES. En el mismo sentido, son relevantes estas cuestiones porque la VIII Asamblea del Consejo Federal de Educación de la República Argentina (CFE), en el año 2007 mediante la Resolución $N^{\circ} 30$, establece que la producción de saberes sobre la enseñanza, la formación y el trabajo docente, formará parte de las funciones del Sistema de Formación Docente, ampliando así sus funciones. Es desde estos supuestos políticos que la tesis de referencia se planta, ¿desde qué supuestos epistemológicos y metodológicos el Sistema Formador debe desarrollar esta función?

Sin embargo, si bien la producción de saberes es un rasgo de identidad del Sistema de Formación Docente establecido por la normativa vigente, estos saberes no suelen ser reconocidos como tales, es decir, no son identificados como formas valiosas de respuesta a problemas relevantes en el ámbito de las políticas públicas y no circulan, o circulan poco, tanto en el ámbito educativo, académico y científico. Al mismo tiempo que, los aspectos vinculados a las condiciones de producción y validación de estos conocimientos han sido poco considerados y estudiados, constituyéndose en una de las áreas de vacancia de la Educación Superior y de las Ciencias de la Educación. Ahora bien, este hecho tiene al menos dos consecuencias:

a) el Sistema Educativo no puede beneficiarse del examen, la crítica y la reformulación de las prácticas posibles cuando los conocimientos toman estado público y;

b) las trayectorias profesionales de los formadores no capitalizan estos desarrollos pues, la falta de instancias donde dichos conocimientos circulen disminuye las oportunidades de los profesores de realizar actividades académicas reconocidas como parte de su desarrollo profesional. 
Revista de la Escuela de Ciencias de la Educación, año 13, nRo. 12, vol. 2, julio a diciembre de 2017. Páginas 67-86. ISSN 1851-6297. ISSN 2362-3349 (EN LINEA). EL SISTEMA DE FORMACIÓN DOCENTE Y LA PRODUCCIÓN, RELEVANCIA, VALIDACIÓN y Legitimación del Conocimiento. Algunas dimensiones para abordar una agenda aún pendiente. Cristina Rafaela Ricci

Es así que las consideraciones anteriores habilitan el planteo de interrogantes en torno a cómo es la relación producción/transmisión del conocimiento en la formación docente y acerca de cómo se visibiliza y jerarquiza la producción de saberes y de conocimiento en el marco del Sistema Formador. Asimismo, permiten interrogarse sobre cómo, dónde y a través de qué procedimientos, el conocimiento producido en las instituciones formadoras se valida: ¿Es desde el campo académico? ¿Es desde el propio Sistema Formador? ¿Son las escuelas las que lo validan, en la medida en que encuentren en estas investigaciones herramientas de intervención para los problemas vinculados con la enseñanza y con la educación? ¿Cuáles son los procesos y mecanismos que se ponen en juego en el proceso de investigación que permiten justificar-validar resultados, conclusiones y hallazgos? La sola presentación del informe final de investigación, su evaluación y difusión, ¿habilitan a que estos resultados, conclusiones y hallazgos sean considerados conocimiento científico? ¿Desde qué perspectivas epistemológicas y metodológicas hay que analizar las investigaciones que se desarrollan en los IES, en general y en los Institutos Superiores de Formación Docente (ISFD) en particular?

En todo caso, retomando la perspectiva epistemológica de la tesis, lo cierto es que en la primera década del siglo XXI van cobrando fuerza las críticas a los planteos epistemológicos y metodológicos de la concepción heredada de la modernidad, al mismo tiempo que se percibe la emergencia de otros sujetos y de otros modos de producción y validación del conocimiento. Es por ello que, cuando se quiere analizar la producción y legitimación de saberes y conocimientos en los IES y en los ISFD es necesario pensar más allá de los cánones modernos, enfatizando la dimensión institucional y social de la ciencia considerada como una actividad que se desarrolla en múltiples ámbitos o contextos y por diversos sujetos.

Ahora bien, otra racionalidad, ¿es posible?

Considerando que sí es posible, la hipótesis de trabajo de la tesis sostiene que los planteos epistemológicos y metodológicos de la línea fundadora o concepción heredada de la modernidad no son aptos para otorgar estatus científico a los saberes y conocimientos producidos en los IES y en los ISFD. En cambio, los aportes de epistemologías y metodologías emergentes a partir de la segunda mitad del siglo XX ofrecen elementos para repensar críticamente los discursos, las definiciones y las praxis institucionales en relación con la producción, validación, legitimación y relevancia de estos saberes y conocimientos. En cuanto a la racionalidad que operaría en la producción de saberes y conocimiento en los IES y en los ISFD, la misma estaría más vinculada a una racionalidad colectiva, colaborativa, contextual e inductiva que a una racionalidad formal, es decir, hipotético-deductiva. 


\section{Dimensión político-educativa}

Hasta fines del año 2006 los ISFD eran considerados como "institutos terciarios", como "instituciones de transición" entre las escuelas secundarias y las universidades, algo así como instituciones "cenicientas" del sistema educativo y "aplicacionistas" de saberes producidos por otros. Como quedó planteado en la introducción, con la sanción de la LEN en ese mismo año, se instituye a los IES como "parte constitutiva del nivel de Educación Superior y tiene como funciones, entre otras, la formación docente inicial, la formación docente continua, el apoyo pedagógico a las escuelas y la investigación educativa" (LEN, 2006, art. 72).

Ahora bien, concebir a la formación docente como sistema implica que las acciones tendientes a la cobertura de las necesidades de formación se planifiquen a escala del sistema nacional y jurisdiccional, y no necesariamente a escala de cada institución. Esta consideración es importante porque no siempre será posible que cada jurisdicción y/o institución tenga la totalidad de la oferta de formación que se requiere para los recursos humanos del sistema educativo, ciertas especialidades cuentan con escasos formadores calificados en el país y por ello el expediente formal de abrir una carrera específica no asegura la posibilidad de ofrecerla con personal adecuado. Contar con formadores especializados lleva tiempo y requiere políticas de promoción específicas (Cfr. Resolución $\mathrm{N}^{\circ} 30$, Anexo I, punto 31). Esto mismo es aplicable a la producción de conocimiento situado.

Sin embargo, todos sabemos que ninguna normativa por sí misma cambia la realidad por lo tanto, ¿Cómo puede modificarse en el presente el pasado institucional de los IES y en particular la de los ISFD? ¿Cuál es el escenario institucional en el que se produce este pasaje de "terciario" a "superior"? ¿Cómo afecta la crisis de la transmisión cultural y el declive de las instituciones a este cambio de perspectiva en relación con los IES/ISFD? La crisis de la enseñanza, ¿Es sólo un problema pedagógico y didáctico? Considerar a la enseñanza como un problema político, ¿Sería una de las transformaciones contemporáneas que configurarían otro tipo de sistema escolar, de formación docente y de producción de conocimiento?

La LEN establece que, el INFD es uno de los organismos responsables de impulsar y desarrollar acciones de investigación en el ámbito de los IES/ISFD. En el mismo sentido el Consejo Federal de Educación señala que la formación de docentes comparte con el conjunto de la docencia cuatro rasgos:

1. El estatus profesional de la docencia es particular.

2. Se trata de una profesión masiva.

3. La docencia es una actividad internamente diferenciada en cuanto a los saberes que se requieren para su ejercicio y en cuanto a las funciones que desempeñan sus profesionales.

4. La docencia es una profesión que hace de los conocimientos y de la 
Revista de la Escuela de Ciencias de la Educación, año 13, nRo. 12, vol. 2, julio a diciembre de 2017. Páginas 67-86. ISSN 1851-6297. ISSN 2362-3349 (EN LINEA). EL SISTEMA DE FORMACIÓN DOCENTE Y LA PRODUCCIÓN, RELEVANCIA, VALIDACIÓN y Legitimación del Conocimiento. Algunas dimensiones para abordar una agenda aún pendiente. Cristina Rafaela Ricci

transmisión cultural su sentido sustantivo, pero guarda una relación peculiar con tales conocimientos y con los saberes.

La formación de docentes comparte con el conjunto de la docencia estos cuatro rasgos, pero responde al último de ellos de manera singular. Si en el ejercicio usual del trabajo docente el saber sobre la transmisión no suele tematizarse, en el caso de la formación docente es su objeto central pues es, en torno al saber sobre la enseñanza y al trabajo docente que se estructuran los procesos de formación. Encontramos aquí un rasgo de identidad del sistema formador. Este es un asunto respecto del cual no puede ser sustituido por ninguna otra instancia institucional. Entonces es propia del sistema formador la producción de saberes y de conocimiento sobre la enseñanza, sobre el trabajo docente y sobre la formación. Se trata de un rasgo de identidad cuyas potencialidades son enormes pero que ha sido poco considerado al menos en la historia reciente del sistema educativo.

En este sentido, es interesante contrastar lo que sucede con los saberes sobre la enseñanza en los ámbitos que tienen propósitos académicos clásicos y en los que tienen propósitos de formación docente. Mientras que los estudios académicos especializados aún no dilucidan la naturaleza del conocimiento presente en los procesos de toma de decisiones de los docentes y acerca de la clase de conocimientos que se requieren para enseñar, la formación inicial tiene en las instancias de práctica y residencia experiencia acumulada en la identificación de los saberes sobre la enseñanza y en relación con la producción de un trabajo especializado sobre la formación. A su vez, en las instituciones formadoras están cada vez más desarrollados dispositivos que proponen a los docentes en formación situaciones para que puedan reflexionar sobre sus decisiones, para que puedan analizar sus resultados, para que consideren las dificultades afrontadas y para que evalúen posibles alternativas para sus prácticas (Resolución $\mathrm{N}^{\circ} 30$, Anexo I, puntos 36 y 37). Sin embargo, Gary Anderson y Kathryn Herr (2007) sostienen que aún queda pendiente la discusión sobre la potencialidad de la investigación desarrollada por los formadores de formadores para reconstruir la base de conocimientos públicos sobre procesos de enseñanza y de aprendizaje, así como sobre la gestión escolar.

En relación con esto, Pedro Enríquez (2004), plantea que la producción de conocimiento efectuado por los docentes implicados en el proceso pedagógico es lo que va a permitir la aprehensión profunda de la compleja y cambiante realidad educativa. Por su parte, Violeta Guyot (2011) sugiere un modelo para la investigación que incluya al mismo tiempo la teoría y la práctica, así como la epistemológica y la pedagógica para analizar las condiciones de posibilidad de un conocimiento riguroso de las prácticas docentes y del conocimiento en su complejidad y sus consecuencias para la investigación educativa. Al mismo tiempo resulta sugestiva su pregunta "¿Cómo investigamos la educación?" de acuerdo a los objetos que se podrían ir delimitarlo en tan vasto campo, de sus 
condiciones históricas de posibilidad, de los instrumentos críticos para operar en la producción y en la legitimación de conocimientos. Según Guyot (2011), la cuestión epistemológica requiere una atención especial pues el conocimiento aparece como preocupación en un doble registro: por el condicionamiento que implica la propia perspectiva epistemológica para la efectiva realización de las prácticas investigativas y, por la estrecha relación entre el conocimiento y la educación presente en el fenómeno educativo mismo puesto que, sin conocimiento no hay educación posible. Lo que nos remite a la segunda dimensión: la dimensión epistemológica y metodológica.

\section{Dimensión epistemológico-metodológica}

La concepción de conocimiento científico y su correlato metodológico heredados del paradigma moderno está hoy en crisis.

En relación con esto, en primer lugar Eric Hobsbawm (1994) sostiene que "ningún otro período de la historia ha sido más impregnado por las ciencias naturales-positivas, ni ha sido más dependiente de ellas que el siglo XX. No obstante, ningún otro período "se ha sentido menos a gusto con ellas" (Hobsbawm, 1994, p.516). A grandes rasgos esto es así porque los recelos y temores hacia la ciencia se vieron alimentados por cuatro sentimientos: la ciencia era incomprensible; sus consecuencias, ya fuesen prácticas o morales, eran impredecibles y probablemente catastróficas; la ciencia ponía de relieve la indefensión del individuo y, que la ciencia minaba la autoridad. Sin olvidar el sentimiento de que la ciencia era intrínsecamente peligrosa, en la medida en que interfería en el orden natural de las cosas (Hobsbawm, 1994).

En segundo lugar, Thomas Kuhn (1962) que para algunos teóricos inicia con su obra La estructura de las revoluciones científicas la era de la posciencia, cuestiona al positivismo y sostiene que la ciencia no progresa hacia fin alguno como la verdad. Lo que ocurre más bien es que las teorías evolucionan desde formas anteriores: las teorías no se corresponden con una realidad definitiva, dada de una vez para siempre sino que tanto las teorías como la realidad se construyen. Esta postura estaba muy alejada de los supuestos de la filosofía vigente hasta la segunda mitad del siglo XX cuando Kuhn consideraba irrelevantes las normas metodológicas universales y ponía en cuestión la existencia de una racionalidad general por encima de los compromisos tácitos de las comunidades científicas. En este mismo sentido, Antonio Gutiérrez (2000) plantea que este cambio de enfoque se centró en la eliminación de la barrera que presentaban los dos contextos clásicos vinculados a la ciencia moderna, el de justificación y descubrimiento, lo cual posibilitó la irrupción de la historia de la ciencia como fuente de argumentos y desarrollos teóricos. Por su parte, Trejo (2010) sostiene que con Kuhn (1962) se asiente que los cambios de paradigmas reflejan, entre otras cosas, cambios de época y, por lo tanto, cambios de cosmovisiones lo que remite a la imposibilidad de sostener 
Revista de la Escuela de Ciencias de la Educación, año 13, nRo. 12, vol. 2, julio a diciembre de 2017. Páginas 67-86. ISSN 1851-6297. ISSN 2362-3349 (EN LINEA). EL SISTEMA DE FORMACIÓN DOCENTE Y LA PRODUCCIÓN, RELEVANCIA, VALIDACIÓN

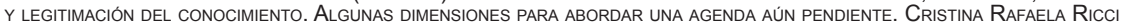

la presencia de una estructura fija y única en la construcción de conocimiento. Las variantes son múltiples y posibles lo cual desmitifica la validez del pensamiento único y del método universal, se constata una realidad cada vez más compleja y multidimensional, llena de eventos y acciones, interacciones y retoracciones que no admiten racionalismos reductivos.

Por su parte Paul Feyerabend (1975), al afirmar que la idea de un método único, de una racionalidad fija que surgen de una visión del hombre demasiado ingenua, es insostenible abre la puerta a un pluralismo metodológico donde deberían buscarse propuestas alternativas para la producción de conocimiento, llegando a proponer un procedimiento contra-inductivo basado en la contradicción sistemática de teorías y resultados experimentales bien establecidos y a aumentar el contenido empírico con la ayuda del principio de proliferación. Para esto sostiene que el científico debería ser heterodoxo y proponer ideas contrapuestas: se debe proponer ideas distintas recurriendo para ello, como fuente de inspiración, a lo que haga falta, incluso a teorías antiguas y desechadas, sin que importe para nada que hayan sido "falsadas empíricamente» en su tiempo o que, probablemente, tengan orígenes metafísicos, religiosos o míticos. La idea es buscar sistemas conceptuales que choquen con los datos experimentales aceptados e, incluso, proponer nuevas formas de percepción del mundo hasta entonces ignoradas. El científico haría uso de cuanto tenga a la mano: sugerencias heurísticas, concepciones del mundo, disparates metafísicos, restos y fragmentos de teorías abandonadas, entre otras formas posibles.

En este punto, sostengo que los planteos de Kuhn y de Feyerabend se presentan como puntos de quiebre y bisagra epocal y, al mismo tiempo, como la posibilidad para pensar epistemologías y metodologías alternativas a la moderna, articuladas con la historia y con el contexto socio-cultural e institucional. Si bien reconozco, junto con Gregorio Klimovsky (2006) que, estos planteos

no llegaron a constituir una posición unificada que pudiera oponerse de manera frontal a la sustentada por los hipotético deductivistas (...) Kuhn, imprimió un giro copernicano a la epistemología contemporánea y la desplazó de su posición logicista tradicional a otra de carácter más sociologista y vinculada con la historia de la ciencia (...) mientras que, Feyerabend critica la manera tradicional de diseñar el método hipotético deductivo y la noción de teoría (...) e insiste, como también lo hicieron Kuhn y otros epistemólogos a su manera, en que no existen datos empíricos independientes de las teorías científicas, defendiendo la no unicidad del método científico (Klimovsky, 2006, pp. 341; 380-381).

Asimismo, coincido con Elizabeth Padilla (2009) cuando considera que, los esfuerzos de Kuhn y Feyerabend entre otros, no esbozaron una doctrina monolítica reconocible como alternativa al modelo anterior o a la filosofía neopositivista de la ciencia que se ha apoyado en una teoría del significado de corte 
verificacionista pero lograron, sin embargo, provocar modificaciones en el desarrollo de la epistemología actual que ha llevado a reconocer la necesidad de incorporar procedimientos y estrategias conceptuales distintas a las tradicionales. Esto ha producido lo que se denomina "giro interpretativo", con consecuencias tanto para reformular planteos epistemológicos como para elaborar nuevos modos de descripción del quehacer científico. Una de las consecuencias consiste en atribuirle al fenómeno interpretativo un debilitamiento de la inteligibilidad. Otra consecuencia, de mayor alcance heurístico, ve en el giro interpretativo la condición para acceder a una visión más completa y compleja de los procesos cognitivos en ciencia (Padilla, 2009). Sin embargo, Morin considera que, "(...) aún somos ciegos al problema de la complejidad -siendo que- las disputas epistemológicas entre Popper, Kuhn, Lakatos, Feyerabend lo pasan por alto" (Morin 1997, pp. 34-35). Por lo que Morin insiste en la necesidad de que estos planteos requieren ser complementados con la construcción de un pensamiento complejo y un paradigma de la complejidad.

En este punto, cuando Michael Gibbons (1994) se aboca a la discusión de los atributos de un nuevo modo de producción de conocimiento, en contraposición al modelo tradicional de producción de conocimiento de carácter disciplinario al que llama Modo 1, que conlleva un complejo de ideas, métodos, valores y normas que deben ser adoptadas en la producción, legitimación y difusión del conocimiento para que el mismo tenga el carácter convencional de científico, propone el Modo 2 de producción de conocimiento, al que define como un conjunto de prácticas cognitivas y sociales, que tiene características propias. En este punto coincido con la crítica realizada por Gibbons (1994) en relación con su propuesta de considerar otro modo de producción de conocimiento porque entiendo, junto con él, que el Modo 1 está caracterizado fundamentalmente por la investigación disciplinaria institucionalizada en las universidades, mientras que el Modo 2 tiene, entre sus principales características, la transdisciplinariedad, la heterogeneidad y la flexibilidad. En el Modo 2 emergen nuevas formas organizacionales donde los grupos de investigación están menos institucionalizados, la gente se reúne en equipos temporales y en redes que se disuelven cuando el problema es resuelto o redefinido. A pesar de que los problemas son pasajeros y los grupos son de corta vida, el patrón de organización y comunicación persiste como una matriz, a partir de la cual, futuros grupos y redes dedicados a problemas diferentes pueden conformarse (Gibbons, 1994; 1998).

Por su parte, Jaime Jiménez Guzmán (2010) sugiere otras vías alternativas, no formales, locales y regionales de acceso al conocimiento para lo cual desarrolla lo que denomina Modo 3, al que conceptualiza como un modo de producción de conocimientos distinto del Modo 1 y del Modo 2. La diferencia de los Modos 1 y 2 con el Modo 3, sostiene Jiménez Guzmán (2010), radica en que el Modo 3 se dedica a temas de investigación que están directamente 
Revista de la Escuela de Ciencias de la Educación, año 13, nRo. 12, vol. 2, julio a diciembre de 2017. Páginas 67-86. ISSN 1851-6297. ISSN 2362-3349 (EN LINEA). EL SISTEMA DE FORMACIÓN DOCENTE Y LA PRODUCCIÓN, RELEVANCIA, VALIDACIÓN y Legitimación del Conocimiento. Algunas dimensiones para abordar una agenda aún pendiente. Cristina Rafaela Ricci

asociados a resolver necesidades sentidas por la comunidad, es decir, es socialmente responsable frente a la problemática de su entorno inmediato vinculado a las necesidades sociales actuales.

Ahora bien, Gibbons además de criticar al Modo 1 de producción de conocimiento, heredado de la modernidad, agudiza la crítica y la extiende a los contextos de descubrimiento y de justificación, critica anticipada ya por Kuhn (1962). Esta crítica, puede complementarse con las propuestas de Javier Echeverría (1995) y de Esther Díaz (2000) acerca de los contextos propios de la actividad científica. Por su parte Echeverría (1995) plantea que la actividad científica puede desarrollarse en cuatro contextos: el contexto de educación, el contexto de innovación, el contexto de evaluación y el contexto de aplicación. Si bien presenta los cuatro contextos separados, esto es sólo con fines analíticos, ya que destaca la profunda interacción que se realiza entre ellos: no hay educación para la ciencia sin innovaciones, ni innovaciones sin aplicación, ni educación, innovación o aplicación sin valoraciones en todos y cada uno de los contextos. Sin embargo, estos contextos tal como los elabora Echeverría (1995), aun cuando tiene en cuenta elementos que van mucho más allá de la simple validación formal exigida por la epistemología tradicional, siguen sosteniendo que la ciencia es el modo por excelencia de producción de conocimiento. En cambio Esther Díaz (2007, 2010, 2013), si bien coincide con esta propuesta de Echeverría (2010) de pasar de los dos contextos clásicos de producción y validación del conocimiento a estos cuatro, va más allá y propone habilitar lo que denomina epistemología ampliada y modulación metodológica.

Esta perspectiva propuesta por Díaz sugiere que en la actualidad se trata de considerar no sólo el proceso racional de invención de teorías y su posible (y discutible) justificación, sino que también hay que incluir la dimensión institucional y social de la ciencia como actividad que se desarrolla en múltiples ámbitos o contextos. En tal sentido sugiere que otra racionalidad es posible porque, simplemente se trata de pensar desde otro lugar, no irracional sino racional pero histórico, encarnado, constituido desde las prácticas y los discursos y no desde idealizaciones cuasi platónicas. Se trata, asimismo, de rescatar el concepto de verdad, pero no de una verdad intemporal sino consensuada según criterios sociales, culturales, epocales $\mathrm{y}$, fundamentalmente, surgida desde los dispositivos de poder, que también son dispositivos de verdad. Existen, por lo tanto, criterios distintos que pueden garantizar la validez de los discursos y de las prácticas investigativas a partir del plexo de sentidos, distintos y vigentes en cada comunidad histórica (Díaz, 2007).

Ahora bien, reconsiderando la normatividad propia de la actividad científica, Díaz plantea que, desde cierto punto de vista, la misma posee un carácter meramente técnico o instrumental (a la manera de un imperativo hipotético), pero 
que sólo adquiere pleno sentido en función de los objetivos que la orientan. Estos objetivos no son meramente epistémicos, sino que pertenecen también al ámbito de la ética y de la política. Estos objetivos se construyen en el marco de las diferentes comunidades históricas y no únicamente en el de la comunidad científica la que forma parte de la primera, en las cuales se producen estrategias de poder relacionadas con la circulación de los discursos considerados verdaderos o que producen efectos de verdad (Díaz, 2007, 2010 y 2013).

En este punto, y teniendo en cuenta el tema abordado en este artículo y en la tesis, es importante poner en evidencia la tensión entre la jerarquía del estatus del conocimiento producido en los IES/ISFD al que muchos catalogan como "no-científico", sin tener en cuenta que una de las especificidades de este conocimiento es que tiene relación con saberes de la práctica y con saberes construidos en la acción e interacción con otros en el contexto de las instituciones educativas.

Ahora bien, frente a la diversidad de perspectivas y enfoques en relación con las categorías conocimiento-saber, investigación-enseñanza, adhiriendo al posicionamiento de Gloria Edelstein (2013) y a los aportes de Elena Achilli (2001) considero que, tanto los docentes como los investigadores producen saberes y conocimientos de distinto tipo, por lo que hay que distinguir entre la lógica de producción de conocimiento y la lógica de uso y de enseñanza del mismo, infiriéndose entonces que no hay una jerarquización de actividades y posiciones, sino una necesaria discriminación entre ellas. A partir de la diferenciación entre investigación, en tanto producción de conocimiento y, el saber producido por el profesor sobre su enseñanza es posible introducir otra diferencia entre investigación, formación y práctica reflexiva siendo que esta última se relaciona con el ejercicio profesional, es decir, con la comprensión del propio quehacer. En el caso de la formación, esta se vincula con el propósito excluyente de que los futuros docentes internalicen tanto actitudes como habilidades propiciatorias del análisis permanente de su prácticas. En este punto Edelstein marca que,

es preciso superar la relación planteada en términos dilemáticos entre sensibilidad experiencial [saber] y la indagación teórica [conocimiento]. Si interesa el conocimiento de las prácticas, reconociendo las particularidades de cada situación concreta, no se pueden obviar las necesarias relaciones entre el bagaje intelectual, fruto de la propia experiencia y el saber público de la ciencia y las artes disponibles. Es en este punto en que juegan un papel fundamental los procesos reflexivos entendidos como comprensiones situacionales. Los docentes construyen en forma permanente su propio conocimiento, cuando se posicionan en esta suerte de conversación tanto con la situación como con los presupuestos subjetivos que orientan su pensamiento y su acción en cada escenario concreto. A ello hay que integrar, sin duda, el conocimiento que, generado en otros tiempos y espacios, sirve de soporte teórico, 
Revista de la Escuela de Ciencias de la Educación, año 13, nRo. 12, vol. 2, julio a diciembre de 2017. Páginas 67-86. ISSN 1851-6297. ISSN 2362-3349 (EN LINNEA). EL SISTEMA DE FORMACIÓN DOCENTE Y LA PRODUCCIÓN, RELEVANCIA, VALIDACIÓN y Legitimación del Conocimiento. Algunas dimensiones para abordar una agenda aún pendiente. Cristina Rafaela Ricci

permitiendo al docente nuevas construcciones de orden conceptual desde las cuales interpretar y comprender las problemáticas de situaciones de la práctica objeto de análisis (Edelstein, 2013, pp. 45-46).

Profundizando el análisis de la relación entre investigación y formación docente, junto con Achilli considero importante realizar una primera aproximación a esta relación en un doble sentido: "Por un lado, [resulta necesario] acotar y precisar el/los significado/s con que usualmente usamos cada noción. Por el otro, acotar y precisar el/los significado/s con que construí[mos] la relación entre ambos campos" (Achilli, 2001, p. 17).

En un sentido general, entendemos por investigación, "al proceso por el cual se construyen conocimientos acerca de alguna problemática de un modo sistemático y riguroso" (Achilli, 2001, p. 20). En cambio, por formación docente entendemos "un determinado proceso en el que se articulan prácticas de enseñanza y de aprendizaje orientadas a la configuración de sujetos docentes/enseñantes" (Achilli, 2001, pp. 22-23). A partir de esta categorización de 'formación docente' resulta clave la noción de 'práctica docente' en un doble sentido: "Por un lado, como práctica de enseñanza que supone cualquier proceso formativo y, por otro, como la apropiación del mismo oficio magisterial, de cómo iniciarse, perfeccionarse y/o actualizarse en la práctica de enseñar" (Achilli, 2001, p. 23). Ahora bien, de un modo general la práctica docente alude a una práctica desarrollada por sujetos cuyo campo identitario se construye alrededor de los procesos fundantes del quehacer educativo como son los procesos de enseñanza y de aprendizaje. De hecho, estos procesos suponen determinados modos de circulación de conocimientos. Sin embargo puede diferenciase este núcleo fundante de la escolaridad -en sentido amplio- como práctica pedagógica para referirse a la práctica que se despliega en el contexto del aula caracterizada por la relación docente, alumno (grupo-clase) y conocimientos, en un determinado contexto. En cambio, la noción de práctica docente se construye a partir de la práctica pedagógica pero la trasciende al implicar, además, "un conjunto de actividades, interacciones, relaciones que configuran el campo laboral del sujeto maestro o profesor en determinadas condiciones institucionales y sociohistóricas" (Achilli, 2001, p. 23).

Precisados algunos términos nodales, ahora sí puede caracterizarse la relación entre investigación y formación docente como una "relación problemática", según la califica Achilli:

tanto la investigación como la formación docente -en su carácter de práctica pedagógica- tienen en común el trabajo con el conocimiento. En este sentido, el conocimiento se configura en el campo de intersección entre los procesos de investigación -ámbito en el que se generan/construyen- y los procesos derivados de la práctica docente -ámbito de re-trabajo al interior de los procesos de enseñanza y de aprendizaje- (Achilli, 2001, p. 26). 
En la investigación, sostiene Achilli, se realiza un trabajo metódico y reflexivo en la generación de nuevos conocimientos sobre un determinado campo disciplinario. En la formación/práctica docente también se produce un trabajo metódico y reflexivo sobre el conocimiento. Sin embargo, es un trabajo centrado, fundamentalmente, en torno a los criterios de la acción pedagógica con los que se pondrá en circulación determinado campo de conocimientos. De ahí que los objetivos y la lógica que orientan uno u otro quehacer son diferentes. En el caso de la investigación los objetivos y la lógica están orientados por el proceso de construcción de una problemática de investigación. En el caso de la docencia los objetivos y la lógica están orientados por el proceso de construcción de una problemática pedagógica. De ahí que se puede decir que, "las prácticas docentes y de investigación remiten a oficios diferentes. Tener en cuenta que se trata de prácticas conformadas desde lógicas de diferentes oficios resulta relevante porque (...) permite entender la complejidad de cada una en su especificidad" (Achilli, 2001, p. 28).

En este punto el conocimiento producido en los ISFD bien puede ser considerado "como campo de intersección de la práctica de investigación y la práctica docente -en su especificidad de práctica pedagógica-" (Achilli, 2001, p. 33), asumiendo diferentes modos relacionales. De entre los modos relacionales posibles, destacaré, siguiendo a Achilli, tres en los que pueden estar inmersos los docentes en sus prácticas de enseñantes que tienen como trasfondo las articulaciones entre dicha práctica y los procesos de investigación o de generación de conocimientos, si bien es importante no entender estas modalidades relacionales como clasificaciones del conjunto de prácticas docentes, sino como grandes tendencias o como construcciones claves -en el sentido de una abstracción que acentúa ciertos aspectos parciales de una problemática compleja-, "con la intención de re-marcar y explicitar con más claridad los condicionamientos que limitan a esta práctica. Por ende, no remite a situaciones 'individuales'; más bien parte de entender a los sujetos y a sus prácticas como sociales" (Achilli, 2001, p.35).

La primera tendencia hace referencia a un modo relacional recursivo, complejo o dialéctico con el conocimiento en el que los docentes participan tanto de la producción/generación de un campo de conocimientos a través de procesos de investigación, como de la transposición didáctica en tanto, circulación de esos conocimientos mediante los procesos de enseñanza y aprendizaje. El segundo modo relacional es el de apropiación/internalización dialéctica del conocimiento que es el que caracteriza a una práctica docente desarrollada en torno al trabajo sobre el conocimiento involucrándose activamente en la internalización de un objeto o campo de conocimiento. Por último, está el modo relacional enajenado que implica no sólo una disociación de ese trabajo de internalización del docente, sino también una profunda disociación, entre los conocimientos generados en el campo de la investigación y aquellos conocimientos que circulan en el aula (Achilli, 2001). 
Revista de la Escuela de Ciencias de la Educación, año 13, nRo. 12, vol. 2, julio a diciembre de 2017. Páginas 67-86. ISSN 1851-6297. ISSN 2362-3349 (EN LINEA). EL SISTEMA DE FORMACIÓN DOCENTE Y LA PRODUCCIÓN, RELEVANCIA, VALIDACIÓN y Legitimación del Conocimiento. Algunas dimensiones para abordar una agenda aún pendiente. Cristina Rafaela Ricci

\section{Para seguir reflexionando}

La temática que aborda y problematiza la tesis presentada en este artículo para su reflexión y debate, tiene una doble relevancia. La primera está vinculada con la política educativa en cuanto a la producción de saberes y conocimiento socialmente relevante, haciendo foco en los ISFD como instituciones productoras de estos saberes y conocimiento, institución que con el conjunto de las instituciones escolares en todos los niveles del sistema educativo, hoy están en declive y requieren transformaciones, donde el desafío radica en re-institucionalizar la escuela como institución socio-político-cultural mediante la producción de saberes y de conocimiento. La segunda es de corte epistemológico y metodológico, vinculada a la ciencia moderna, también en declive y sujeta a las transformaciones que en la posmodernidad se están produciendo en ella, que plantea como desafío reinstitucionalizar los ámbitos, modos y sujetos productores de saberes y de conocimientos.

Asimismo, cabe señalar que la cultura científica construida desde el siglo XVII hasta nuestros días ha incorporado la visión de orden y progreso como leyes de la naturaleza y de la historia de la humanidad. Paradójicamente, esas leyes fundadas en un tiempo lineal y unidimensional ha conducido a sostener en nuestros días la idea de 'fin de la historia', es decir, la cancelación del futuro y la impotencia del ser humano para transformar un mundo que, en la perspectiva determinista, se presenta como dado para siempre (Guyot, 2011). Así, una nueva visión de la ciencia que permita incorporar la complejidad, haría posible establecer otros vínculos con la naturaleza y con la sociedad, vínculos en los que el hombre recupere su condición de sujeto constructor y creador del conocimiento sin excluirse a sí mismo del conocimiento, generando un nuevo tipo de dialogo del hombre, en tanto sujeto, con la naturaleza, con otros hombres y consigo mismo. La reducción de lo complejo a lo simple, la disyunción entre la cultura científica y la cultura de las humanidades fueron, señala Guyot (2011), operaciones epistemológicas que pretendían garantizar el rigor y la objetividad del conocimiento, pero el resultado ha sido la incapacidad de pensar las totalidades, los conjuntos, aislándose lo objetos de sus contextos.

Al mismo tiempo, cabe destacar que el giro de la epistemología ha introducido de lleno la cuestión de la práctica y del sujeto en la consideración del conocimiento científico: nuevas opciones epistemológicas y metodológicas afectan al conocimiento científico y a las prácticas del conocimiento como denomina Guyot (2011) a las prácticas investigativas, a las prácticas docentes y a las prácticas profesionales, entre otras, y tienen efectos en la producción de conocimientos y en la configuración de subjetividades. La posibilidad de intervención en la modificación de las propias prácticas pone de relieve la importancia de los modos en que los sujetos se relacionan con el conocimiento a la hora de investigar, de enseñar o de ejercer una profesión. Por lo cual, coincidimos con Guyot (2011) cuando plantea algunas hipótesis de trabajo y para la reflexión que quedan aquí reseñadas. 
En primer lugar la hipótesis que indica que las opciones epistemológicas -y metodológicas agregamos- determinan, o al menos condicionan, la producción e interpretación de las teorías e impactan en las prácticas investigativas, docentes y profesionales, entre otras. La segunda hipótesis sostiene que la epistemología -y la metodología completamos- abordada en su articulación con la historia de la ciencia permite pensar y recrear críticamente las prácticas del conocimiento en un campo disciplinar actualizado. Por último, la hipótesis que considera que el paradigma de la complejidad permitiría reformular el campo epistemológico de las ciencias humanas para investigar, intervenir y transformar las prácticas investigativas, las prácticas docentes y las prácticas profesionales atendiendo a las complejidades específicas que cada una de ellas plantean en relación a los sujetos y al conocimiento, a las instituciones, a los regímenes de prácticas y al sistema social en el que se concretan. Estas consideraciones habilitan un replanteo de la concepción de ciencia (Guyot, 2011).

Finalmente, hacemos nuestras algunas cuestiones y señalamientos planteados en el Anexo I de la Resolución N 30 del CFE (2007). ¿Cómo se resuelve la relación producción/reproducción del saber y del conocimiento en la formación docente y, cómo podría resolverse en dirección a visibilizar y jerarquizar la producción de saberes y de conocimiento en el marco de sistema formador?

Al respecto, en primer lugar, se hace necesario definir condiciones de funcionamiento del sistema formador que habiliten a los formadores para el desarrollo de situaciones formalizadas de producción de saberes y de conocimiento directamente vinculados con la complejidad de formar docentes, habilitando nuevas formas de relación con el saber y con el conocimiento sobre la formación y sobre la tarea de enseñar. En segundo lugar, parece promisorio explorar formas de articulación entre las instituciones formadoras y las escuelas, en las que se generen procesos de circulación, evaluación y validación de innovaciones en el terreno de la enseñanza.

Los desarrollos teóricos compartidos pueden ser considerados como algunos apuntes, que bien pueden contribuir para comenzar a reflexionar sobre la real posibilidad de producción de conocimiento por parte de los ISFD a partir de lo que denomino perspectivas epistemológicas y metodológicas emergentes, no alternativas a la mirada hegemónica y homogénea de la modernidad porque si fuesen alternativas marcarían la pervivencia a la concepción heredada de la modernidad. Al mismo tiempo pueden aportar a la resignificación de los procesos socio-históricos de construcción de conocimientos localizados, tanto desde lo geográfico como desde lo cultural e institucional habilitando otras miradas y voces que, desde las grietas que resquebrajan la modernidad, están produciendo conocimiento y saberes, en tanto metáforas de la realidad.

Estas perspectivas emergentes dan cuenta de que en el horizonte y en el escenario epocal comienzan a vislumbrase otras racionalidades, otros sujetos, otras instituciones que, como los IES y los ISFD tienen que asumir su 
Revista de la Escuela de Ciencias de la Educación, año 13, nRo. 12, vol. 2, julio a diciembre de 2017. Páginas 67-86. ISSN 1851-6297. ISSN 2362-3349 (EN LINEA). EL SISTEMA DE FORMACIÓN DOCENTE Y LA PRODUCCIÓN, RELEVANCIA, VALIDACIÓN

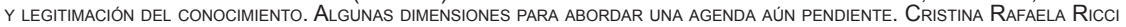

rol y responsabilidad en un campo de producción, validación y legitimación de saberes y conocimientos fundamentales para la transmisión, recreación y creación de la cultura.

\section{Referencias bibliográficas}

- Achilli, E. (2001). Investigación y Formación Docente. Rosario: Laborde Editor.

- Anderson, G. y Herr, K. (2007). El docente-investigador: Investigación - Acción como una forma válida de generación de conocimientos. En I. Sverdlick, (Comp.) (2007).La investigación educativa. Una herramienta de conocimiento y de acción. Buenos Aires: Novedades Educativas. Editorial. Consultado el 19 de mayo de 2015 Disponible en:http://books.google.com.ar/books?id=9ZQvkLwlaGwC\&printsec=frontcover\&hl=es\&source=gbs_ge_summary_r\&cad $=0 \# v=$ onepage $\& \& \&=$ false

- Arroyo, B. (2011). La teoría fundamentada. Una aproximación a la generación de teorías [educiencri.blogspot.com] Recuperado de: http://educiencri.blogspot.com/2011/05/ la-teoria-fundamentada-una-aproximacion.html

- Bardin. L. (1996). El análisis de contenido. Madrid: Ediciones Akal.

- Consejo Federal de Educación de la República Argentina (2007). Resolución № 30 y Anexo I. Hacia una Institucionalidad del Sistema de Formación Docente en Argentina.

- de la Torre, G.(2008). Teoría Fundamentada y MCC. Madrid: Universidad Autónoma de Madrid.

- Díaz, E. (2000). La posciencia. El conocimiento científico en las postrimerías de la modernidad. Buenos Aires: Biblos.

- Díaz, E. (2007). Entre la tecnociencia y el deseo. La construcción de una epistemología ampliada. Buenos Aires: Biblos.

- Díaz, E. (2013). La investigación habitada por devenires. Ponencia presentada en el XII Seminario Internacional de Filosofía: Nietzsche/Deleuze, en la Universidad Estadual de Campinas (Unicamp), Brasil, 2013, publicado por dicha institución. En la presente versión se forma un nuevo pliegue -el epistemológico- insertando fragmentos de "Hacia una multiplicidad metodológica", Perspectivas metodológicas, № 13, Buenos Aires, UNLa, 2013, y elaborando conceptos ad hoc.

- Díaz, E. y Rivera, S. (2000). La actividad científica y su insoportable carga ética. En Díaz, E. (2000). La posciencia. El conocimiento científico en las postrimerías de la modernidad. Buenos Aires: Biblos.

- Díaz, E. (2010). La construcción de una metodología ampliada.Vol.6 no.3, Universidad Nacional de Lanús sep./dic. 2010, versión on-line:revistasaludcolectiva@yahoo.com.ar

- Echeverría, J. (1995). Filosofía de la ciencia. Madrid: Akal.

- Edelstein, G. (2013). Formar y formarse en la enseñanza. Buenos Aires: Paidós.

- Enriquez, P. (2004). Modalidades y discusiones en torno a la noción de docente investigador. Universidad Católica de Córdoba, REDUC. Consultado el 19 de marzo de 2015. Disponible en: http://www.uccor.edu.ar/paginas/REDUC/romero.enriquez.pdf.

- Feyerabend, P. (1975). Tratado contra el método. Madrid: Técnos.

- Foucault, M. (1981). El orden del discurso. Barcelona: Tusquets. 
- Gibbons, M. (1998). Pertinencia de la educación superior en el siglo XXI. Paris: UNESCO.

- Gibbons, M. (1994). La nueva producción del conocimiento. La dinámica de la ciencia y la investigación en las sociedades contemporáneas. Barcelona: PomaresCorredor.

- Gutiérrez, A. (2000). Cerca de la revolución: la biología en el siglo XXI. En Díaz, E. (2000). La posciencia. El conocimiento científico en las postrimerías de la modernidad (pp. 109-124). Buenos Aires: Biblos.

- Guyot, V. (2011). Las prácticas del conocimiento. Un abordaje epistemológico: educación, investigación y subjetividad. Buenos Aires: Lugar Editorial.

- Hobsbawm, E. (1994). Historia del Siglo XX. Buenos Aires: Crítica.

- Jiménez Guzmán, J. et al., Vías alternas, no formales, locales y regionales de acceso al conocimiento: el caso del Centro para la Innovación y Desarrollo Educativo (CIDE) mexicano. Ponencia presentada en las VIII Jornadas Latinoamericanas de Estudios Sociales de la Ciencia y Tecnología. Buenos Aires, Argentina, julio de 2010.

- Kuhn, T. (1990 [1962]). La estructura de las revoluciones científicas. México: Fondo de Cultura Económica.

- Klimovsky, G. (2006). Las desventuras del conocimiento científico. Una introducción a la epistemología. Buenos Aires: AZ Editores.

- Ley $N^{\circ} 26.206$ de Educación Nacional.

- Morin, E. (1997). Introducción al pensamiento complejo. Barcelona: Gedisa.

- Padilla, E. (2009). Contribución de la interpretación a la epistemología, con especial referencia al aporte de Kuhn. Revista En-claves del pensamiento, 3 (6), 15-24.

- Pardo, R. (2000). Verdad e historicidad. El conocimiento científico y sus fracturas. En Díaz, E. (2000). La posciencia. El conocimiento científico en las postrimerías de la modernidad (pp.37-62). Buenos Aires: Biblos.

- Pievi, N. (2009). Documento metodológico orientador para la investigación educativa. Buenos Aires: Ministerio de Educación de la Nación.

- Soneira, A. (2009). La Teoría fundamentada en los datos (Grounded Theory) del Glaser y Strauss. En: I. Vasilachis de Gialdino, (coord.) (2009). Estrategias de investigación cualitativa. Barcelona: Gedisa.

- Trejo, M. (2010).Teologías y construcción de conocimiento. Entre sujetos y disciplinas. En González de Cardedal, O. (2010). Nuevos horizontes (pp 139-164). Buenos Aires: Editorial San Benito.

- Vázquez Sixto, F. (1996). El análisis de contenido temático. Objetivos y medios en la investigación psicosocial. (Documento de trabajo). (pp. 47-70). Universitat Autónoma de Barcelona. 